\title{
THE RELATIONS OF THE PNEUMOGASTRIC AND OTHER NERVE FIBRES TO THE PERSISTENT ARTERIAL ARCHES.a
}

\author{
By ALEC FRASER, M.B., F.R.C.S.; \\ Professor of Anatomy, R.C.S.I.
}

[Commanicated to the Section of Anatomy and Physiology. January 26, 1906.]

THE remarks which I have to make are based mainly upon what can be easily dissected on the left side of the human subject. The superior laryngeal represents the nerve which passes to the larynx between the internal carotid and the early dorsal aorta. The latter disappears between the carotid and the aortic or fourth arterial arch, and so lets the nerve free. Should the early connection persist as a cord, the nerve would be found at the junction formed between it and the internal carotid artery, just as the recurrent or inferior laryngeal nerve is always found looping round the junction of the ligamentum arteriosum with the dorsal aorta. Between the internal carotid and the ligamentum arteriosum-which latter represents the sixth arterial arch-there are two arterial arches, one of which, the aortic, has always a complete loop of nerve fibres around it, formed mainly by the upper cardiac branches of the vagus, that of the left side passing in front of the arch to join its neighbour of the right side behind the arch. The primitive fifth arterial arch disappears; but there is clear evidence of a second loop of nerve fibres round the aortic arch coming chiefly from the last cervical and first dorsal ganglia of the sympathetic, those from the left side

a This communication was illustrated by drawings on the blackboard. 
being mainly in front and joining those from the right side behind. As I am only dealing with the persistent arches, I may leave this loop out of consideration for the present. In addition to those enumerated-viz., superior laryngeal round the internal carotid arch, the cardiac branch loop round the aortic arch, the evidence of a second loop round the aorta, which may represent that of the lost fifth arterial arch, the recurrent laryngeal round the sixth arterial arch, I invariably find another loop formed from the vagus, passing round the front aspect of the functional pulmonary artery (on the left side) to join the nerves behind. This loop is quite clear of the branches running to the lung root, and points suggestively to the Gill cleft origin of the lung. 